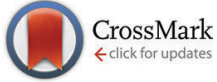

Cite this: Phys. Chem. Chem. Phys., $2016,18,2112$

Received 10th November 2015, Accepted 4th December 2015

DOI: $10.1039 / \mathrm{c} 5 \mathrm{cp} 06858 \mathrm{~d}$

www.rsc.org/pccp

\section{Physical insights into salicylic acid release from poly(anhydrides) $\dagger$}

\author{
Queeny Dasgupta, ${ }^{a}$ Kaushik Chatterjee ${ }^{a b}$ and Giridhar Madras*ac
}

Salicylic acid (SA) based biodegradable polyanhydrides (PAHs) are of great interest for drug delivery in a variety of diseases and disorders owing to the multi-utility of SA. There is a need for the design of SA-based PAHs for tunable drug release, optimized for the treatment of different diseases. In this study, we devised a simple strategy for tuning the release properties and erosion kinetics of a family of PAHs. PAHs incorporating SA were derived from related aliphatic diacids, varying only in the chain length, and prepared by simple melt condensation polymerization. Upon hydrolysis induced erosion, the polymer degrades into cytocompatible products, including the incorporated bioactive SA and diacid. The degradation follows first order kinetics with the rate constant varying by nearly 25 times between the PAH obtained with adipic acid and that with dodecanedioic acid. The release profiles have been tailored from $100 \%$ to $50 \%$ SA release in 7 days across the different PAHs. The release rate constants of these semi-crystalline, surface eroding PAHs decreased almost linearly with an increase in the diacid chain length, and varied by nearly 40 times between adipic acid and dodecanedioic acid PAH. The degradation products with SA concentration in the range of 30-350 ppm were used to assess cytocompatibility and showed no cytotoxicity to HeLa cells. This particular strategy is expected to (a) enable synthesis of application specific PAHs with tunable erosion and release profiles; (b) encompass a large number of drugs that may be incorporated into the PAH matrix. Such a strategy can potentially be extended to the controlled release of other drugs that may be incorporated into the PAH backbone and has important implications for the rational design of drug eluting bioactive polymers.

\section{Introduction}

Poly(anhydrides) (PAHs) are a versatile class of polymeric biomaterials that have found extensive use in biomedical applications, particularly in the area of drug delivery. ${ }^{1-3}$ These polymers are best suited for short term drug release; ${ }^{4,5}$ however, the controlled release of several drugs has been successfully achieved by loading drugs in the PAHs. ${ }^{6,7}$ These polymers are typically hydrophobic but they possess hydrolytically labile anhydride linkages, which qualify these materials as prospective biodegradable materials. The major benefit of PAHs is that they are typically composed of diacid precursors. Because these precursors are often endogenous to the human body, PAHs do not elicit inflammatory responses when used as biomaterial implants. ${ }^{8,9}$

\footnotetext{
${ }^{a}$ Centre for Biosystems Science and Engineering, Indian Institute of Science, Bangalore-560012, India. E-mail: giridhar@chemeng.iisc.ernet.in; Tel: +91-80-2293 2321

${ }^{b}$ Department of Materials Engineering, Indian Institute of Science, Bangalore-560012, India

${ }^{c}$ Department of Chemical Engineering, Indian Institute of Science, Bangalore-560012, India

$\dagger$ Electronic supplementary information (ESI) available. See DOI: 10.1039/c5cp06858d
}

A variety of PAH structures has been synthesized and evaluated to accomplish application specific drug release behavior. Apart from changing the nature/structure of the polymeric backbone to aliphatic or aromatic, combinations of the two have also been tried. ${ }^{10,11}$ PAHs like poly(maleic anhydride) have been synthesized such that anhydride linkages are present in the pendant side chains instead of the primary backbone. ${ }^{12,13}$ Owing to the diverse requirements of stimuli-responsiveness ${ }^{14}$ in drug release applications, it is important to understand the correlation between the structure of the polymer and its subsequent influence on physical properties such as degradation and drug release. ${ }^{15}$

Aliphatic PAHs have created significant interest in the area of short term drug release, particularly because of their simple diacid constitution. They are semi-crystalline in nature and typically have melting temperatures higher than the physiological temperature. ${ }^{16}$ These are commonly synthesized by simple solvent-free melt polycondensation techniques and may, therefore, be considered safe for biomedical applications. Since PAHs come under the class of insoluble, hydrophobic polymers, degradation or mass loss involves an inevitable step of erosion (as a consequence of polymer chain scission). For such hydrolytic chain scission to occur, the bonds present in the vicinity of the cleavable bonds also play a 
pivotal role. A more hydrophobic chemical structure present in the proximity of the anhydride bond will allow less water retention and subsequently, slower degradation than in a hydrophilic structure.

The influence of linker structure on release from the $\mathrm{PAH}$ backbone has been studied previously. ${ }^{17}$ However, a systematic study of the degradation and release properties of a family of SA based PAHs have not been conducted. In the present study, we use the particular strategy of varying the chain length of diacid to understand its influence on the degradation/erosion kinetics and on the SA release behavior. This study thus provides a systematic comparison of SA-loaded polyanhydrides that offers new physical insights into changes that occur in physicochemical, degradation and release properties, resulting from the differences in chain length of the reactant. We believe that these physical insights can facilitate the rational design of SA loaded polymers for drug delivery.

SA is a potent drug used for its antibacterial, ${ }^{18,19}$ antiinflammatory, ${ }^{20,21}$ anti-pyretic, and anti-cancerous ${ }^{22}$ applications. However, the dosages vary significantly depending on the intended application. The time course of release is also an important characteristic that determines its suitability for an application. The PAHs are of particular interest in drug delivery because they undergo surface erosion and are most suitable for providing controlled release. The anti-inflammatory and anti-microbial effects of SA are well reported and the immediate release of SA from these anhydrides could help mitigate these issues. The only parameter varied in these PAHs is the diacid such as adipic acid, suberic acid, sebacic acid and dodecanedioic acid. These are linear, aliphatic diacids that have monotonically increasing chain lengths.

\section{Experimental}

\subsection{Materials}

Diacyl chlorides, adipoyl chloride (AC, Sigma Aldrich), suberoyl chloride (SC, Sigma Aldrich), sebacoyl chloride (SeC, TCI chemicals, India), and dodecanedioyl chloride (DC, Sigma Aldrich) were used. Acetic anhydride (AAn) was procured from Sigma Aldrich. Salicylic acid (SA) was obtained from SD Fine Chemicals, India. Solvents used at various stages of the study include ethyl acetate (EtoAc), chloroform, dichloromethane (DCM), dimethylsulfoxide (DMSO), $N, N$-dimethylformamide (DMF), and acetone (all from Merck, India).

\subsection{Synthesis}

Step I: synthesis of SA based diacids. SA (1 mol. equiv. $7 \mathrm{~g}$, $50.7 \mathrm{mmol})$ was dissolved completely in THF $(10 \mathrm{~mL})$. Pyridine ( 2 mol. equiv., $8.3 \mathrm{~mL}, 101.4 \mathrm{mmol}$ ) was added and allowed to stir in this solution for $15 \mathrm{~min}$ at $35{ }^{\circ} \mathrm{C}$. The respective diacyl chlorides ( 0.5 mol. equiv.) were added slowly at $0{ }^{\circ} \mathrm{C}$ (ice bath) to the stirred solution. The ice bath was removed after addition and the reaction mixture was stirred for 12 to $16 \mathrm{~h}$ at $37{ }^{\circ} \mathrm{C}$. After completion of the reaction, hydrochloric acid was added to the reaction mixture kept at $0{ }^{\circ} \mathrm{C}$ (ice bath) to precipitate the diacid. Acid was added until a $\mathrm{pH}$ of 2 was reached. The obtained precipitate (white) was washed repeatedly with water. The precipitate was then vacuum filtered and dried to constant weight before further reaction with anhydride in the next step. This step was based on previously reported study. ${ }^{23}$

Step II: synthesis of acetylated diacids. $5 \mathrm{~g}$ of the synthesized diacid was placed in a round bottomed flask with excess AAn $(50 \mathrm{~mL})$ at $160{ }^{\circ} \mathrm{C}$ under reflux conditions for $2.5 \mathrm{~h}$. After this, the excess AAn was removed from the system at $85{ }^{\circ} \mathrm{C}$ under vacuum. The obtained precipitate was dissolved in THF and then precipitated by pouring this solution drop-wise into stirred petroleum ether at $4{ }^{\circ} \mathrm{C}$. The activated diacids were acetylated.

Step III: polymerization of acetylated diacid. The anhydride precursors were polymerized by a simple melt condensation process. The precursors obtained in the previous step were melted at $160{ }^{\circ} \mathrm{C}$ and stirred under $\mathrm{N}_{2}$ atmosphere and vacuum (60 $\mathrm{mm} \mathrm{Hg}$ ) for $\sim 1 \mathrm{~h}$. The obtained polymer was dissolved in THF and dried in a rotary evaporator at $37{ }^{\circ} \mathrm{C}$. The synthesis scheme is detailed in scheme 1 . The resulting PAHs were named P4ASA, P6ASA, P8ASA and P10ASA, respectively, wherein the numbers indicate the number of methylene units in the respective diacids, PA indicates poly(anhydrides) and SA indicates salicylic acid.

The loading concentrations of SA as obtained by dissolving polymer discs in PBS ( $\mathrm{pH}$ 9.0) were 4.6, 3.5, 2.6 and $1.9 \%$ by weight for P4ASA, P6ASA, P8ASA and P10ASA, respectively.

\subsection{Materials characterization}

2.3.1 FTIR spectroscopy. The synthesized PAHs were chemically characterized by FTIR spectroscopy. Spectra were obtained on a Perkin-Elmer Frontier FT-NIR/MIR spectrometer under universal attenuated total reflectance (UATR-FTIR) mode. The recorded range extended from 4000 to $650 \mathrm{~cm}^{-1}$ and the spectrum was an average of 32 scans with a resolution of $4 \mathrm{~cm}^{-1}$.

2.3.2 ${ }^{1}$ H NMR spectroscopy. Proton-nuclear magnetic resonance (NMR) spectroscopy was also used for chemical characterization. The spectra were obtained on a $400 \mathrm{MHz}$ Bruker Avance NMR spectrometer. $2 \mathrm{mg}$ of the prepolymer was dissolved in $500 \mu \mathrm{L}$ of $\mathrm{CDCl}_{3}$ (for P4ASA and P6ASA) and d6-DMSO (for P8ASA and P10ASA) with $0.03 \%(\mathrm{v} / \mathrm{v})$ tetramethylsilane as the internal reference (Deutero, Germany).

2.3.3 Molecular weight determination. The molecular weight distribution (MWD) was obtained by gel permeation chromatography (GPC, Waters, Milford, USA). The setup was maintained at $50{ }^{\circ} \mathrm{C}$ and consisted of an isocratic pump, three size-exclusion columns (Waters Styragel HR 4, HR 3, and HR 0.5 columns $(300 \times 7.5 \mathrm{~mm}))$, a differential refractive index detector (Waters, R415) and data acquisition system. The eluent used in the system was THF and was run at a flow rate of $0.9 \mathrm{~mL} \mathrm{~min}^{-1}$. Samples were injected into a Rheodyne valve with a $100 \mu \mathrm{L}$ sample loop. The chromatograph was converted to MWD using the universal calibration with polystyrene standards (Polymer Lab, Poole, UK). The Mark-Houwink constants were obtained from previously reported literature. ${ }^{24}$ 
<smiles>[X]C(CCC(C)(C)C(=O)Cl)C(=O)Cl</smiles><smiles>CC(=O)OC(C)=O</smiles><smiles>CC(=O)OCO</smiles><smiles>CC(=O)OC(O)c1ccccc1OC(=O)CCCCC(=O)Oc1ccccc1C(O)O</smiles>

Acetylated Adipoyl Diacid<smiles>CC(C)C(=O)OC(O)c1ccccc1OC(=O)CCCCC(=O)Oc1ccccc1C(O)OC(=O)C(C)(C)C</smiles>

P4ASA

Scheme 1 Synthesis scheme of SA incorporated PAHs (P4ASA). Diacyl chlorides were changed to result in different PAHs.

2.3.3 Differential scanning calorimetry. Synthesized PAHs were thermally characterized on a differential scanning calorimeter (DSC, TA Instruments, Q2000). PAH samples weighing 5-7 mg were placed in a copper pan and crimped. The samples were then exposed to a uniform temperature program from -50 to $200{ }^{\circ} \mathrm{C}$ with a temperature ramp of $5{ }^{\circ} \mathrm{C} \mathrm{min}^{-1}$.

2.3.4 Surface water wettability. The wettabilities of the samples were analyzed by measuring the water contact angle with a contact angle goniometer (Dataphysics). The static contact angles were evaluated by placing a $1 \mu \mathrm{L}$ droplet of ultrapure water (Sartorius) on polymer discs (10 $\mathrm{mm}$ in diameter). The contact angle was measured after the drop equilibrated $\sim 10 \mathrm{~s}$ after it was placed. The data are represented as mean \pm standard deviation (S.D.) based on three independent measurements.

2.3.5 Hydrolytic degradation studies. The samples were melted and made into discs of $5 \mathrm{~mm}$ diameter, which were then placed in $20 \mathrm{~mL}$ phosphate buffered saline (PBS) of $\mathrm{pH}$ 7.4. They were maintained at $37{ }^{\circ} \mathrm{C}$ in an incubator shaker under shaking conditions (100 rpm), and subsequently removed from PBS at specified time points and weighed after drying to constant weight in a vacuum desiccator. The mass loss of the polymer was measured using the following formula:

$$
\% \text { weight loss }=\frac{M_{0}-M_{\mathrm{t}}}{M_{0}} \times 100
$$

In eqn (1), $M_{0}$ and $M_{\mathrm{t}}$ indicate the initial and final weights of the PAH discs, respectively. All samples were made in triplicate and the mass loss data are represented as mean \pm standard deviation.

2.3.6 SA release studies. SA release from the $\mathrm{PAH}$ matrices was evaluated to compare the release kinetics of the different polymers. Discs, as prepared for degradation studies, were immersed in $20 \mathrm{~mL}$ PBS at $37{ }^{\circ} \mathrm{C}$ and $100 \mathrm{rpm}$ and allowed to degrade over time. The release media were collected and the UV spectra were obtained using a UV-Vis spectrophotometer (Shimadzu, UV-1700 PharmaSpec). SA has characteristic absorption maxima at $297 \mathrm{~nm}$. The SA concentration was determined by comparison with the standard calibration curve.

2.3.7 Cytocompatibility studies. HeLa, a cervical carcinoma cell line, was used for assessing the cytocompatibility of the PAHs. HeLa cells are a sturdy cell line classified as a standard cell line for evaluation of cytocompatibility of biomedical polymers. The cells were cultured in Dulbecco's modified Eagle's medium (DMEM, Gibco, Life Technologies) supplemented with $10 \%$ (v/v) fetal bovine serum (FBS, Gibco, Life Technologies) and antibiotics $(1 \%(\mathrm{v} / \mathrm{v})$ penicillin-streptomycin). The cells were given growth conditions of $37{ }^{\circ} \mathrm{C}$ and $5 \% \mathrm{CO}_{2}$. This study used cells of Passage 13. Cytocompatibility was estimated using an indirect method wherein cells were treated with conditioned media. Conditioned media were prepared by immersing $5 \mathrm{~mm}$ discs (thickness $1 \mathrm{~mm}$ ) of P4ASA, P6ASA, P8ASA and P10ASA, and allowing them to undergo hydrolytic degradation for $24 \mathrm{~h}$ in $5 \mathrm{~mL}$ of complete media (DMEM + FBS). HeLa cells were exposed to this pre-conditioned media and allowed to grow in its presence. Cell viability was monitored at specific time points of 1 day and 3 days. The initial cell seeding density was 5000 cells per well in a 48-well plate. The cells were first allowed to attach to wells for $6 \mathrm{~h}$ (in the presence of fresh medium). The fresh medium was then replaced with $500 \mu \mathrm{L}$ per well of conditioned media. This study used the fresh medium as the control. Both fresh and conditioned media were replenished after every $24 \mathrm{~h}$ during the course of the study.

After treatment in conditioned media, cell viability was estimated using MTT (3-(4,5-dimethylthiazol-2-yl)-2,5-diphenyltetrazolium Bromide, Sigma Aldrich) assay. The respiratory chain reduces MTT and other tetrazolium salts by the action of oxidoreductases produced by live cells. A stock solution of $5 \mathrm{mg} \mathrm{mL} \mathrm{m}^{-1}$ of MTT was made initially. The working solution 
was a diluted solution of $1 \mathrm{mg} \mathrm{mL} \mathrm{mL}^{-1}$. For the cell proliferation assay, $200 \mu \mathrm{L}$ of MTT solution $\left(1 \mathrm{mg} \mathrm{mL} \mathrm{mL}^{-1}\right)$ was added to each well. The well plates were kept unperturbed at $37{ }^{\circ} \mathrm{C}$ in $5 \% \mathrm{CO}_{2}$ for $3 \mathrm{~h}$. Water insoluble formazan crystals were solubilized in DMSO and the absorbance was measured at $570 \mathrm{~nm}$ in a well plate reader (Synergy HT, Biotek). The absorbance value is proportional to the number of mitochondria in the well. The mean of absorbance values from five independent wells was obtained to give the final absorbance. The data are represented as mean \pm S.D. for $n=5$. Analysis of variance (ANOVA) with Tukey's test was used for all statistical analysis, and significances were considered significant for $p<0.05$.

2.3.8 Anti-bacterial activity. To assess the pharmacological activity of the drug released from the PAHs, the effectiveness of the polymers were evaluated against $S$. aureus (ATCC 25923). Luria Bertani (LB) broth medium was used to culture $S$. aureus. The bacteria were allowed to grow overnight in an incubator shaker $(100 \mathrm{rpm})$ and maintained at a temperature of $37^{\circ} \mathrm{C}$. The overnight culture $(200 \mu \mathrm{L})$ was added to $5 \mathrm{~mL}$ of fresh $\mathrm{LB}$ medium and grown for $2 \mathrm{~h}$. PAHs were dispersed in the LB medium $\left(3 \mathrm{mg} \mathrm{mL}^{-1}\right)$ and the bacterial cultures were added to obtain a final optical density of 0.1 at $600 \mathrm{~nm}\left(\mathrm{OD}_{600}\right)$. Bacterial growth was monitored by obtaining $\mathrm{OD}_{600}$ at $6 \mathrm{~h}$ and $24 \mathrm{~h}$ after initial incubation. The readings from the replicates are reported as mean \pm S.D. for $n=3$.

\section{Results and discussion}

The synthesized PAHs have molecular weights ranging from 8300 to $49000 \mathrm{Da}$ (as shown in Table 1) with a polydispersity index of 1.4. The molecular weights increased as the chain length of the diacid precursor increased.

\subsection{FTIR spectroscopy}

All the acids (AC, SC, SeC and DC) show the characteristic IR peak at $1690 \mathrm{~cm}^{-1}$ due to the $-\mathrm{C}=\mathrm{O}$ stretching of carboxylic chloride. After the synthesis of the diacid in the first step, a peak appeared at $1750 \mathrm{~cm}^{-1}$, indicating the formation of the ester bond at the $-\mathrm{OH}$ group of SA. The peak at $1690 \mathrm{~cm}^{-1}$ was still present, indicating the presence of the free $-\mathrm{COOH}$ group. After the reaction with AAn and subsequent polymerization under vacuum, two peaks were observed at $1815 \mathrm{~cm}^{-1}$ and $1740 \mathrm{~cm}^{-1}$, which are characteristic of two $-\mathrm{C}=\mathrm{O}$ stretching vibrations and indicate the formation of the anhydride bond,

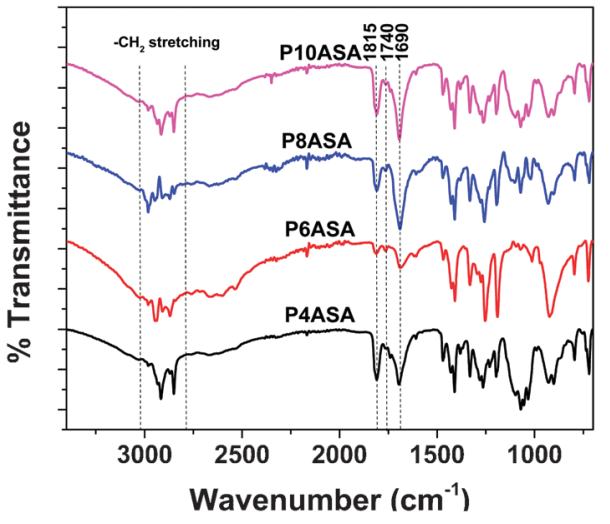

Fig. 1 FTIR spectra of the PAHs.

generally characterized by two peaks (Fig. 1). All peaks match previously reported literature. ${ }^{25}$

\subsection{NMR spectroscopy}

NMR spectroscopy (Fig. S1, see ESI $\dagger$ ) confirmed the chemical characterization as observed in FTIR. All peaks in the ${ }^{1} \mathrm{H}$ NMR spectra match previously reported data in literature. ${ }^{26,27}$ ${ }^{1} \mathrm{H}$ NMR confirmed the presence of all the peaks corresponding to protons in the $-\mathrm{CH}_{2}$ (methylene) units of the diacids. The peaks from the di-carboxylic acids are displayed in the range between 0.8 and $2.4 \mathrm{ppm}$. The peaks of the monomers match those obtained in the polyesters. More shielded $\mathrm{C}_{\gamma}, \mathrm{C}_{\delta}$ protons are present in the higher chain diacids of sebacic and dodecanedioic acid as compared to the lower chain diacids. P4ASA contains protons only attached to $\alpha, \beta$ carbons; whereas, P10ASA contains protons attached to $\alpha, \beta, \gamma, \delta$ and $\varepsilon$ carbons. This confirmed the structure of the PAHs. However, the formation of the anhydride was confirmed by the ${ }^{13} \mathrm{C}$ NMR that confirmed the presence of a peak at $170 \mathrm{ppm}$ corresponding to the carbon of the anhydride bond that was present for all the synthesized PAHs.

\subsection{Differential scanning calorimetry}

Thermal characterization (Fig. S2, see ESI $\dagger$ ) of the four PAHs showed that the melting temperature $\left(T_{\mathrm{m}}\right)$ increases as the chain length of the diacid increases. The $T_{\mathrm{m}}$ values obtained are 53, 57, 66 and $85{ }^{\circ} \mathrm{C}$ for P4ASA, P6ASA, P8ASA and P10ASA, respectively. The longer hydrophobic chains of DC as compared to AC cause the substantial increase of the $T_{\mathrm{m}}$ from 53 to $85{ }^{\circ} \mathrm{C}$. There is also a considerable increase in the crystallization

Table 1 Physical properties of PAHs

\begin{tabular}{|c|c|c|c|c|c|c|c|c|c|c|}
\hline Polyester & $M_{\mathrm{w}}(\mathrm{Da})$ & $T_{\mathrm{g}}{ }^{a}\left({ }^{\circ} \mathrm{C}\right)$ & $T_{\mathrm{m}}{ }^{a}\left({ }^{\circ} \mathrm{C}\right)$ & $T_{\mathrm{c}}{ }^{a}\left({ }^{\circ} \mathrm{C}\right)$ & $\begin{array}{l}\Delta H \\
\left(\mathrm{~J} \mathrm{~g}^{-1}\right)\end{array}$ & $\begin{array}{l}\text { Contact } \\
\text { angle }^{b}\left({ }^{\circ}\right)\end{array}$ & $\begin{array}{l}\text { Mass loss }(\%) \\
\text { in } 7 \text { days }^{c}\end{array}$ & $\begin{array}{l}\text { Degradation } \\
\text { rate constant, } \\
k \times 10^{-3}\left(\mathrm{~h}^{-1}\right)\end{array}$ & $\begin{array}{l}\text { SA release }(\%) \\
\text { in } 7 \text { days }^{c}\end{array}$ & $\begin{array}{l}\text { Release } \\
\text { rate constant, } \\
k_{\mathrm{r}} \times 10^{-3}\left(\mathrm{~h}^{-1}\right)\end{array}$ \\
\hline P4ASA & 8300 & -27 & 53 & 40 & 38 & 32 & 100 & 120 & 100 & 94 \\
\hline P8ASA & 13300 & -5 & 66 & 57 & 92 & 68 & 85 & 11.9 & 84 & 9.9 \\
\hline P10ASA & 49000 & -3 & 84 & 64 & 132 & 82 & 57 & 4.5 & 28 & 2.2 \\
\hline
\end{tabular}

${ }^{a}$ Within a range of $\pm 2{ }^{\circ} \mathrm{C} .{ }^{b}$ Within a range of $\pm 2^{\circ} .{ }^{c}$ Error \pm 1 . 
temperature $\left(T_{\mathrm{c}}\right)$ as the chain length increases. $T_{\mathrm{c}}$ is $40,42,56$ and $74{ }^{\circ} \mathrm{C}$ for P4ASA, P6ASA, P8ASA and P10ASA, respectively. Correspondingly, the $T_{\mathrm{g}}$ ranges from -27 to $-3{ }^{\circ} \mathrm{C}$ and increases with the diacid chain length. The increase in $T_{\mathrm{g}}$ may be attributed to the higher degree of polymerization in the higher chain length PAHs as compared to their shorter chain counterparts (as shown in Table 1). The longer chain restricts the rotational flexibility of the polymer chains, thereby causing an increase in $T_{\mathrm{g}}$. However, since all the synthesized PAHs have $T_{\mathrm{g}}$ lower (and $T_{\mathrm{m}}$ higher) than the physiological temperature $\left(37^{\circ} \mathrm{C}\right)$, this family of PAHs is suitable for biomedical applications. The ends of a polymer chain have more freedom of motion as compared to the inner segments; low molecular weight polymers have more chain ends per unit volume, and therefore, higher free volume and lower $T_{\mathrm{g}}$.

The crystallization temperature $\left(T_{\mathrm{c}}\right)$ also increases as the chain length of the diacid increases. $T_{\mathrm{c}}$ values range from 40 to $74{ }^{\circ} \mathrm{C}$ between the 4 PAHs and increase as a function of the diacid chain length. The degree of crystallinity may be calculated by considering the area under the crystallization curve and is the enthalpy of crystallization $(\Delta H)$. It is observed that the crystallinity increases as the chain length of the diacid increases. The $\Delta H$ values obtained are $38,48,92$ and $132 \mathrm{~J} \mathrm{~g}^{-1}$ for P4ASA, P6ASA, P8ASA and P10ASA, respectively. The increase in crystallinity is generally accompanied with a concomitant increase in the $T_{\mathrm{m}}$, as observed in this family of PAHs.
PAHs with higher loading concentrations of SA $(\sim 15 \%)$ were synthesized and it was observed that these polymers had $T_{\mathrm{m}}$ values very close to $37^{\circ} \mathrm{C}$. This is undesirable as drug delivery is considerably affected if $T_{\mathrm{m}}$ is $\leq 37{ }^{\circ} \mathrm{C}$. Therefore, all further synthesis and characterization were performed on PAHs with loading $<5 \%$.

\subsection{Surface water wettability}

The surface water wettability pertains to the hydrophobicity of the polymeric material. The hydrophobicity increases in the following trend: P4ASA $<$ P6ASA $<$ P8ASA $<$ P10ASA. The values of the water contact angles are tabulated in Table 1 . The increase in the contact angle and subsequent decrease in the wettability may be attributed to the number of methylene $\left(-\mathrm{CH}_{2}\right)$ units present in the parent diacid. Longer aliphatic chains are more hydrophobic than their shorter counterparts. The greater hydrophilicity of the shorter chain diacids may be attributed to the higher loading percentage of SA in these diacids than in their longer chain counterparts. SA is hydrophilic and its greater inclusion in the matrix leads to the increased hydrophilicity of the PAH.

\subsection{Hydrolytic degradation studies}

The hydrolytic degradation rate increases as the chain length decreases and follows the trend P4ASA > P6ASA > P8ASA > P10ASA. P4ASA, P6ASA, P8ASA and P10ASA degrade by $100 \%$,
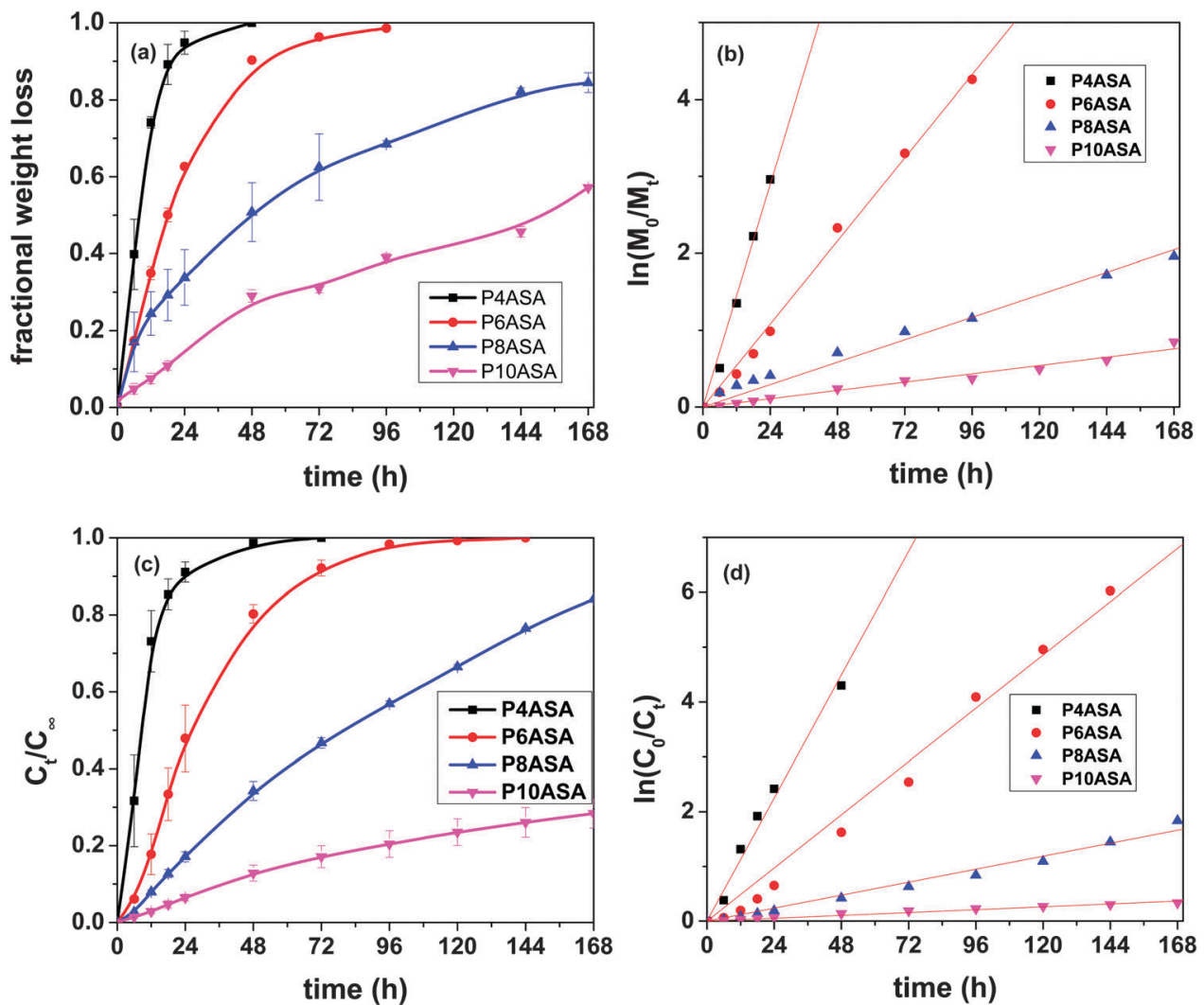

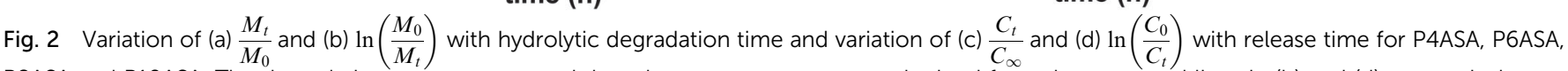
P8ASA and P10ASA. The degradation rate constant and the release rate constant are obtained from the regressed lines in (b) and (d), respectively. 
$90 \%, 50 \%$ and $28 \%$ in $48 \mathrm{~h}$, respectively (Fig. 2a). The degradation data show that the PAHs degrade between $30 \%$ and $100 \%$ in the first $48 \mathrm{~h}$ of hydrolysis. This fast degradation may limit their uses in biomedical applications. However, since the need for antimicrobial, anti-inflammatory drugs is most often immediate after an implantation, and the incorporated SA is reported to possess both these features, they may therefore, be considered suitable for therapeutic applications.

The higher hydrophobic chains of the longer chain acids allow less water permeation than in the shorter chain diacids. The solubility of adipic acid in water is considerably higher than that of the other diacids. ${ }^{28}$ The hydrolytically labile anhydride bonds are located in the proximity of the aliphatic chains. As shown in the contact angle studies, the hydrophobicity of these chains increases with the diacid chain length. Owing to this, the water available at the vicinity of the anhydride bond decreases as the chain length increases. Less water results in lower hydrolysis of the anhydride bonds and consequently, lower mass loss.

Hydrolytic degradation causes chain scission in the backbone of the polymer due to bulk or surface erosion. ${ }^{29,30}$ In most cases, it occurs as a simultaneous bulk or surface phenomenon, wherein one mechanism is more predominant. The anhydride bonds are extremely labile to hydrolysis and thus, the degradation of the surface anhydrides takes place much faster than its penetration into the bulk of the matrix. ${ }^{31}$ Since the mass loss due to degradation is accompanied by a concomitant decrease in the sample dimensions, it is likely that the erosion takes place layer by layer from the surface of the matrix. ${ }^{29}$ This is further corroborated by the kinetics of mass loss, as discussed in the following section.

\subsection{Kinetics of degradation}

The degradation kinetics of these PAHs may be modeled using the following equation:

$$
-\frac{\mathrm{d} M}{\mathrm{~d} t}=k M^{n}
$$

In eqn (2), $k$ is the degradation rate constant and $n$ is the order of degradation. First order release kinetics is followed in all four PAHs as is evident by fitting the degradation data to the following equation:

$$
\ln \left(\frac{M_{t}}{M_{0}}\right)=-k t
$$

In eqn (3), $M_{t}$ and $M_{0}$ are the final and initial weights of the polymer at time $t$ and time $t=0$, respectively. A semi-log plot of $\frac{M_{0}}{M_{t}}$ with time was linearly regressed to obtain the degradation rate constant (Fig. 2b). The values of $k\left(\times 10^{-3} \mathrm{~h}^{-1}\right)$ obtained are 120, 45, 11.9 and 4.5 for P4ASA, P6ASA, P8ASA and P10ASA, respectively. The value of $k$ increases by an order of magnitude for P4ASA as compared to P8ASA and similarly, for P6ASA compared to P10ASA.

\subsection{SA release studies}

SA release was evaluated by monitoring the SA concentration in the release media. It was observed that the release follows the trend P4ASA $>$ P6ASA $>$ P8ASA $>$ P10ASA. In the first $48 \mathrm{~h}$, the polymers P4ASA, P6ASA, P8ASA and P10ASA release 98\%, 80\%, $34 \%$ and $12 \%$, respectively (Fig. 2c). The fast SA release observed in P4ASA and P6ASA may be attributed to two factors: (i) the lesser hydrophobic nature of AC and SC as compared to SeC and DC; (ii) the complete degradation of the P4ASA and P6ASA in $48 \mathrm{~h}$ and $96 \mathrm{~h}$, respectively. Another major factor driving the release of SA from these polymeric matrices is the generally hydrophobic nature of these aliphatic PAHs, ${ }^{29}$ which leads to the tendency for the hydrophilic SA to be released into the media.

\subsection{Kinetics of release}

The kinetics of release followed first order degradation of the $\mathrm{PAH}$ matrices. The SA release may be modeled using the following equation,

$$
-\frac{\mathrm{d} C}{\mathrm{~d} t}=k_{\mathrm{r}} C^{n}
$$

In eqn (4), $C$ is the concentration of the drug remaining in the polymer, $k_{\mathrm{r}}$ is the release rate constant and $n$ is the order of release. Considering first order release and integrating eqn (4), we obtain equation 5 ,

$$
\ln \left(\frac{C_{t}}{C_{0}}\right)=-k_{\mathrm{r}} t
$$

In eqn (5), $C_{0}$ and $C_{t}$ are the initial and final SA concentrations at time $t=0$ and $t=t$, respectively. The semi-log plot of $C_{t} / C_{0}$ with time shows that the $k_{\mathrm{r}}\left(\times 10^{-3} \mathrm{~h}^{-1}\right)$ values obtained are 94, 40, 9.9 and 2.2 for P4ASA, P6ASA, P8ASA and P10ASA, respectively (Fig. 2d). The release rates are consistent with the degradation data and show that the release rate constant increases with a decrease in the diacid chain length. Thus, $k_{\mathrm{r}} / k$ is nearly the same for P4ASA, P6ASA and P8ASA, indicating that release and degradation are related. Similar to the degradation rates, the rate constant of release is nearly an order of magnitude higher for P4ASA as compared to P8ASA. Higher hydrophobicity of P8ASA compared to P4ASA (Table 1) allows lesser water in their vicinity, ${ }^{32,33}$ thereby hindering the degradation and consequently, the release from the PAH matrix. Thus, the degradation of the polymer plays a key role in determining the amount of drug released from the matrix.

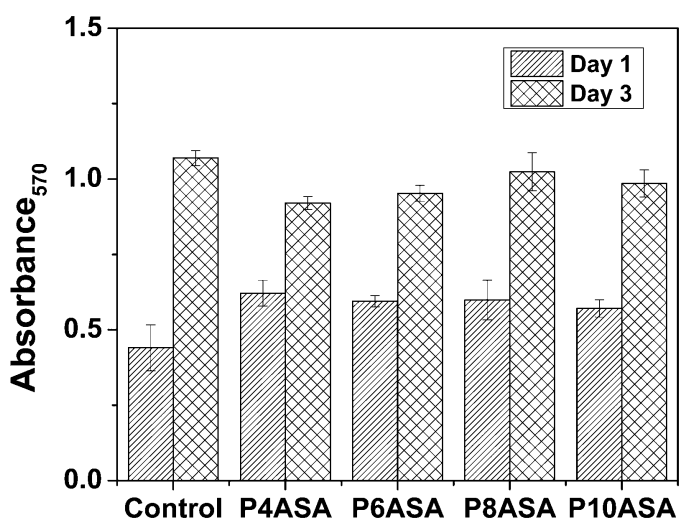

Fig. 3 Cell viability assay (MTT data) of P4ASA, P6ASA, P8ASA and P10ASA based on absorbance at $570 \mathrm{~nm}$. 

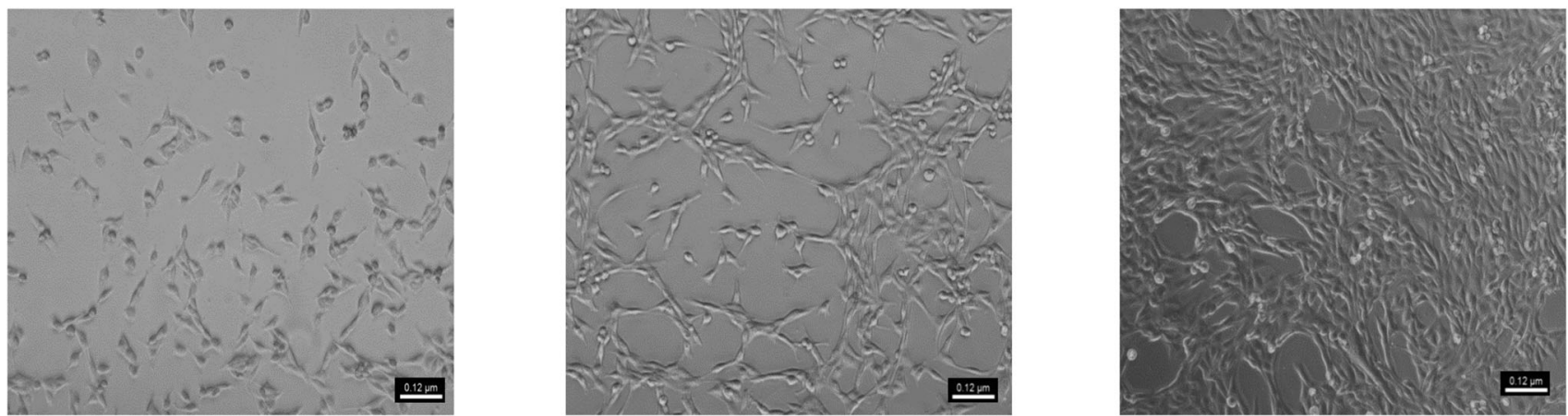

Fig. 4 Optical micrograph of HeLa cells subjected to conditioned medium from P4ASA after (a) $6 \mathrm{~h}$, (b) $24 \mathrm{~h}$ and (c) $72 \mathrm{~h}$.

\subsection{Cytocompatibility studies}

The cell studies showed that cell proliferation was not affected by the degradation products of the PAHs. Despite the decreased $\mathrm{pH}$ of the medium due to the fast degradation of P4ASA and P6ASA, the conditioned media were not toxic to the cells. P4ASA, P6ASA, P8ASA and P10ASA released $350 \mathrm{ppm}, 100 \mathrm{ppm}$, $40 \mathrm{ppm}$ and $25 \mathrm{ppm}$, respectively, in $24 \mathrm{~h}$. These concentrations were estimated as per the release profiles of SA in PBS. Release concentrations may differ slightly in a complete culture medium. Release concentration could not be obtained directly from the medium because of the interference of serum proteins at the maximum emission of SA (297 nm). The MTT assay (Fig. 3) shows comparable cell viability in the conditioned media treated cells to that of the control (fresh medium treated). The cell numbers increased congruously, as monitored after 1 day and 3 days. The cells appeared to be well spread across all samples and good cell to cell contact was established in 3 days (Fig. 4). There was no statistically significant difference observed across the samples.

\subsection{Antibacterial studies}

The antibacterial studies of the PAHs show that all four PAHs are active against $S$. aureus. This study also shows that the released SA is pharmacologically active. The polymers did not show any significant difference from the blank (untreated) after $6 \mathrm{~h}$. However, after $24 \mathrm{~h}$ of growth in the presence of the PAHs, there was a significant drop in the $\mathrm{OD}_{600}$ value indicating

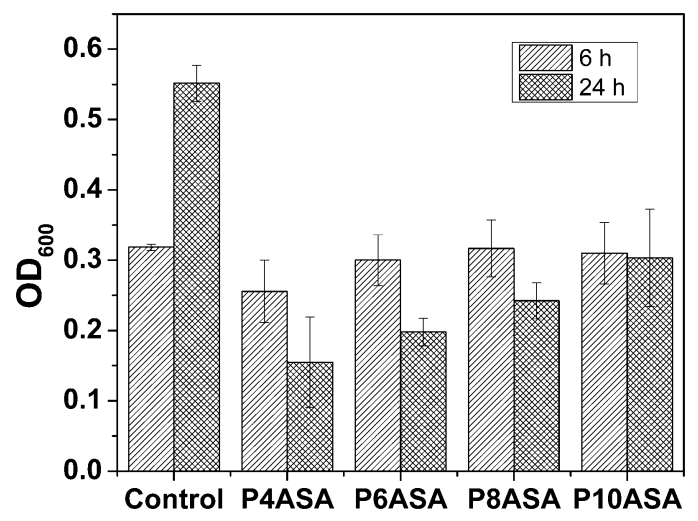

Fig. 5 Antibacterial activity of the PAHs. much less bacterial growth. All the PAHs showed similar antibacterial effect. In order to find significant differences between the polymers, a paired $t$-test with $p=0.05$ was conducted, and it was observed that P4ASA and P10ASA had significantly different antibacterial activities (Fig. 5). This difference may be due to the appreciable disparity in the amount of SA released by the two polymers. P4ASA and P10ASA released 350 and $25 \mathrm{ppm}$, respectively, in $24 \mathrm{~h}$. This higher release of SA from P4ASA may be attributed to its higher activity against $S$. aureus. P4ASA showed $\sim 72 \%$ decrease in bacterial count compared to the control (blank untreated sample).

\section{Conclusions}

We successfully synthesized a family of PAHs for SA delivery. We investigated in detail their degradation and drug release kinetics by varying the chain length of the precursor diacid. The chain length of the precursor plays an important role in controlling the rate of drug release from the polymeric system. It may therefore be effectively used as a strategy to tailor drug release from aliphatic PAHs for application specific purposes. The released drug showed pharmacological activity against bacterial cells. Owing to their cytocompatibility, these SA-based polymers may find uses in biomedical applications.

\section{Acknowledgements}

The authors are grateful to the Department of Biotechnology (DBT), India (BT/PR5977/MED/32/242/2012) for providing financial support for this study. G. M. thanks the Department of Science and Technology (DST), India, for J. C. Bose fellowship. K. C. acknowledges the Ramanujan fellowship from DST. The authors wish to thank the NMR research centre, IISc Bangalore, for providing access to the NMR facility.

\section{References}

1 H. B. Rosen, J. Chang, G. Wnek, R. Linhardt and R. Langer, Biomaterials, 1983, 4, 131-133.

2 K. Leong, B. Brott and R. Langer, J. Biomed. Mater. Res., 1985, 19, 941-955. 
3 W. Khan, V. G. S. Challa, R. Langer and A. J. Domb, Focal Controlled Drug Delivery, Springer, 2014, pp. 3-32.

4 B. D. Ulery, H. M. Kan, B. A. Williams, B. Narasimhan, K. W. H. Lo, L. S. Nair and C. T. Laurencin, Adv. Healthcare Mater., 2014, 3, 843-847.

5 S. Ponnurangam, G. D. O'Connell, C. T. Hung and P. Somasundaran, Colloids Surf., B, 2015, 136, 207-213.

6 J. P. Jain, S. Modi, A. Domb and N. Kumar, J. Controlled Release, 2005, 103, 541-563.

7 J. Wang, G. Yang, X. Guo, Z. Tang, Z. Zhong and S. Zhou, Biomaterials, 2014, 35, 3080-3090.

8 J. E. Vela-Ramirez, J. T. Goodman, P. M. Boggiatto, R. Roychoudhury, N. L. Pohl, J. M. Hostetter, M. J. Wannemuehler and B. Narasimhan, AAPS J., 2015, 17, 256-267.

9 S. S. Snyder, T. J. Anastasiou and K. E. Uhrich, Polym. Degrad. Stab., 2015, 115, 70-76.

10 E. Shen, M. J. Kipper, B. Dziadul, M.-K. Lim and B. Narasimhan, J. Controlled Release, 2002, 82, 115-125.

11 D. Teomim and A. J. Domb, Biomacromolecules, 2001, 2, 37-44.

12 I. Donati, A. Gamini, A. Vetere, C. Campa and S. Paoletti, Biomacromolecules, 2002, 3, 805-812.

13 M. Maniar, X. Xie and A. J. Domb, Biomaterials, 1990, 11, 690-694.

14 S. G. Roy, K. Bauri, S. Pal, A. Goswami, G. Madras and P. De, Polym. Int., 2013, 62, 463-473.

15 A. Göpferich and R. S. Langer, J. Polym. Sci., Part A: Polym. Chem., 1993, 31, 2445-2458.

16 H. R. Kricheldorf and Z. Gomourachvili, Macromol. Chem. Phys., 1997, 198, 3149-3160.

17 A. Prudencio, R. C. Schmeltzer and K. E. Uhrich, Macromolecules, 2005, 38, 6895-6901.
18 N. D. Stebbins, M. A. Ouimet and K. E. Uhrich, Adv. Drug Delivery Rev., 2014, 78, 77-87.

19 L. Rosenberg, A. Carbone, U. Römling, K. Uhrich and M. Chikindas, Lett. Appl. Microbiol., 2008, 46, 593-599.

20 C. Banti, A. Giannoulis, N. Kourkoumelis, A. Owczarzak, M. Kubicki and S. Hadjikakou, J. Inorg. Biochem., 2015, 142, 132-144.

21 R. Amann and B. A. Peskar, Eur. J. Pharmacol., 2002, 447, 1-9.

22 E. Bastiaannet, K. Sampieri, O. Dekkers, A. de Craen, M. van HerkSukel, V. Lemmens, C. van den Broek, J. W. Coebergh, R. Herings and C. van de Velde, Br. J. Cancer, 2012, 106, 1564-1570.

23 Y. Chandorkar, R. K. Bhagat, G. Madras and B. Basu, Biomacromolecules, 2014, 15, 863-875.

24 E. Ron, E. Mathiowitz, G. Mathiowitz, A. Domb and R. Langer, Macromolecules, 1991, 24, 2278-2282.

25 J. P. Coates, Appl. Spectrosc. Rev., 1996, 31, 179-192.

26 B. M. Vogel and S. K. Mallapragada, Biomaterials, 2005, 26, 721-728.

27 E. Mathiowitz, E. Ron, G. Mathiowitz, C. Amato and R. Langer, Macromolecules, 1990, 23, 3212-3218.

28 E. C. Attané and T. F. Doumani, Ind. Eng. Chem., 1949, 41, 2015-2017.

29 A. Göpferich and J. Teßmar, Adv. Drug Delivery Rev., 2002, 54, 911-931.

30 A. Göpferich, Biomaterials, 1996, 17, 103-114.

31 S. Domanskyi, K. L. Poetz, D. A. Shipp and V. Privman, Phys. Chem. Chem. Phys., 2015, 17, 13215-13222.

32 Q. Dasgupta, K. Chatterjee and G. Madras, Biomacromolecules, 2014, 15, 4302-4313.

33 J. Yu, F. Lin, P. Lin, Y. Gao and M. L. Becker, Macromolecules, 2013, 47, 121-129. 